\title{
Proper Nouns in Translation: Should They Be Translated?
}

\author{
Rouhollah Zarei \\ Yasouj University, Iran \\ E-mail: rzarei@yu.ac.ir \\ Somayeh Norouzi \\ Yasouj University, Iran \\ E-mail:somayeh.noroozi61@yahoo.com
}

Received: 04-06-2014

Accepted: 20-07-2014

Published: 01-11-2014

doi:10.7575/aiac.ijalel.v.3n.6p.152

URL: http://dx.doi.org/10.7575/aiac.ijalel.v.3n.6p.152

\begin{abstract}
The translation of proper nouns is not as easy as that of other parts of speech as this is more challenging for certain reasons. The present article presents a descriptive study of proper nouns in translation, scrutinizing the challenges and exploring the solutions. Building on some scholars' approach and suggestions from other researchers, the article clarifies the nature and problems of proper nouns in translation; it seeks to answer three questions: 1) Should proper nouns be translated? 2) What are the problems on the way of translation of the proper nouns? 3) How can the translator overcome such problems? Moreover, strategies applied by the researchers to make their translation easier are also discussed. It follows that translating proper nouns is not simple and there is little flexibility about translating proper nouns.
\end{abstract}

Keywords: proper nouns, translation, strategies

\section{Introduction}

Translation is a special form of communication, aimed at establishing interpretive resemblance between the source text and the target text, governed by the principle of optimal resemblance. Every author would like his/her work to be received and understood by the audience. The word translation comes from the root 'transfer' and thus brings to mind the impression that whatever is in one language is transferable to another one. However, this transference is not easily carried out especially when the subject in question is words with cultural overtones.

Nouns play a key role in understanding a particular text. They have particular features that set them apart from other words. They are deemed critically elemental and essential to writing every piece of text. Basically, nouns are classified as common or proper. Common nouns refer to a class of entities (e.g. cat), while proper nouns have a unique referent (Bill, New York). Proper nouns are part of the linguistic system of the community which may have no meaning in the way that a common name does. There are various points of view, in the sense that some scholars believe proper names carry no meaning, but others say they have meaning like other words. One of the questions which usually addresses the notion of "proper nouns" in any language is whether they should be translated or not. One of the most difficult areas any translator usually faces while translating a text is the translation of proper nouns. Although names are important to human readers, even professional human translators have trouble with names, since the translation of unknown terms still remains a problem. There must be strategies which could be employed to provide the translator with appropriate solutions to this problem.

This article specifically addresses the translation of the proper nouns and aims to explore their construct. The questions addressed in this article are as follows:

1) Should proper nouns be translated?

2) What are the setbacks to the translation of the proper nouns?

3) How can the translator overcome such problems?

Proper nouns are infrequent words relative to other common words. They usually indicate sex, age, history, specific meaning, cultural connotations, animals, companies, festivals, names of persons and geographical places. Aguilera (2008) has called names of persons 'anthroponyms', and geographical places 'toponyms'; the latter is alternatively called 'exonyms' by Nord (2003). Proper nouns may be concrete (objects), figurative (beyond the literal language, metaphorical), technical (scientific names) and slang (used by ordinary people). Many scholars have posed their own definitions of proper nouns. Fernandes (2006) defines proper names as "dense signifiers in the sense that they contain in themselves clues about the destiny of a character or indicates of the way the storyline may develop" (p. 46). Quirk and others (1972) put three certain characteristics for proper nouns as follows:

- Proper nouns do not have the full range of determiners. 
- Proper nouns lack article, such as the name of festivals, months and days of the week, continents, countries, cities, lakes and mountains; exception exist for some classes of proper nouns such as plural names, names of the rivers, seas, hotels, restaurants and newspapers which take definite article.

- Proper nouns are capitalized

Also, the following classification of proper names criteria is established by (Algeo 1973: 9-13):

ORTOGRAPHIC:

- Proper names are capitalized.

MORPHOSYNTACTIC:

- Proper names have no plural forms.

- Proper names are used without articles.

- Proper names do not accept restrictive modifiers.

REFERENTIAL:

- Proper names refer to single unique individuals.

SEMANTIC:

- Proper names do not impute any qualities to the objects designated and are therefore meaningless.

- Proper names have a distinctive form of definition that includes a citation of their expression.

Grammatically, proper nouns behave very much in the same way in the sentence as common nouns. Some proper nouns do not accept demonstrative pronouns as determiners, for instance, nobody can say this John where there are some persons in the name of John. However, it is clearly evident that there are always exceptions and certain limitations on the generalizability of the proper nouns.

Ahanizadeh (2012) states that names may point directly to a single, concrete referent; however, sometimes they may also get a semantic load; therefore, names are mono-referential, which means they refer to a single entity, but they are not mono-functional, since they may have semantic, semiotic, and sound symbolic meanings in literary works. Sperber and Wilson (1986), also, argue about various sorts of proper names. They point out that proper names range from prototypical (names without a descriptive content) to less prototypical cases (a name with a logical entry).

On the whole, whether a given noun is common or proper is not always easy to decide. Also, a given noun may change category depending on how it is used. In the sense that it may be a common noun in one context but a proper noun in another (Sadeghi Ghadi, 2010).

\section{Proper nouns: Change it or leave it?}

Whether a proper noun is real or invented in a language is of great significance in translation. One view is that they do not need to be translated. The reason is that they are not listed in dictionaries so they are not part of our knowledge of the language. Thus, according to Ahanizadeh (2012), proper nouns are treated as labels attached to persons and objects. The opposite view is the description of the referent of the proper noun. It is believed that the strategies used in the translation of proper nouns refer to three different periods:

a) from the twenties to the beginning of the seventies: in this period of time the proper name is considered a grammatical category among others to be translated;

b) a period until the second half of the seventies; and

c) since then until now in which the tendency is the preservation or no translation of proper names.

It is clear that the movement of proper name translation has been from absolute translation to non-translation; it means, currently, translators prefer to preserve the source text original name to avoid probable change in meaning, which means priority of non-translation over translation of proper nouns.

Some scholars believe in transliteration of the proper nouns, in other words making them adapted on the level of spelling, phonology, etc. Others believe in semantic translation-creativity instead of transliteration. According to them as these names may have semantic load, in order to transfer them honestly and accurately, transliteration will not work and it is appropriate is to translate them. There are some geographical areas whose names can be translated into other languages. For instance, the Arabic "Bahrol Ahmar" is translated as "Red Sea" in English and "Daryaye Sorkh" in Persian. Similarly, "the Pacific Ocean" is translated as "Oghiyanoose Aram" in Persian. These names have meanings that preserve the characteristic of the place. Names are indeed translated, if we regard "translation" as a process of linguistic and/or cultural transference. In fiction, authors sometimes invent new names which are fantastic in the source language culture. According to Nord (2003), in some cultures fictional proper names are considered as "culture markers".

Most translation theories agree that the translation of proper names differ in adults' and children's literature.. Jaleniauskienè and Čičelyte (2009) argue about the Harry Potter books which are popular all over the world and include too many proper names - first names and surnames. They have been translated into more than 60 languages and in the first Harry Potter book the number of characters are more than one hundred; since the first audience of these books have been British children, many names are conventional and ordinary to them. They easily recognize foreign or newly coined names but for foreign readers things are not so clear. Therefore, the audience with different cultural and linguistic background have different take on different texts.

Jaleniauskienè and Čičelytė (2009) believe that in real life, proper names usually seem simple meaningless labels signaling reference. For example, the name "Jack" in English serves no meaning; it is just a label for a particular person 
to be identified. They continue proper nouns may be non-descriptive, but they are obviously informative. According to these authors, proper nouns have different aspects in mind: the use of special names, the use of meaningful names, interpretation of names, the contribution to characterization, allusions in proper nouns, text function or effect they create, and so on.

According to some scholars proper nouns which do not have any special meaning should not be translated, while meaningful ones should. As proper nouns are often meaningful, they will be translated. If a name contains clearly recognizable descriptive elements, translators prefer to preserve the descriptive meaning of that name and use literal translation. Applications to proper names are rarely happened, since distortion of an original proper name may replace the intended meaning which may be very far from the origin. If the information is explicit, as in a descriptive name, it can be translated, if the information is implicit, this translation will be ignored, unless the translator intends to compensate it by providing some information (Nord, 2003).

Hirschman and others (2001:21) believe that translation of proper names must ensure that they are

a) not translated when they are personal names;

b) treated idiomatically rather than literally, observing standard naming conventions in the target language; and

c) treated in a format that is usable by target language processing.

Conventions and the format applied in the target language determine how a translator should translate a proper name. On the basis of specific characteristics and the context in which the proper name is used it may be untranslated, or translated with some added definition. Abdolmaleki, (2012) believes that two factors contribute to the translation of proper nouns: purpose of the text and the intended audience. According to him, historically important figures should be translated. For example, "Shah Abbas-e-kabir," in Persian, should be translated as "Shah Abbas, The Great," in English. It is true about acceptable people names like Aristotle and Plato which are translated Arastoo and Aflatoon respectively in Persian. According to Abdolmaleki, (2012), in order to provide an effective translation, it is necessary for the translator to be familiar with characterization as well as literary devices which are used in both languages, for instance, 'Khan', after a name in Persian, connotes greatness. It can be left unchanged if the very word is familiar to the readers, otherwise, it should be translated to 'The Great'. A number of statements by this author, below, clarify the ways proper names can be treated in translation:

1) They can be kept unchanged from the source language text.

2) They can be modified with respect to phonological and graphological system of the target language.

3) They can be expanded in order to provide the information that does not exist in the readers' culture.

4) Even, they can be eliminated if not important to convey the main massage of the text. This way occurs just when the reader confronts confusion if the proper name is noted in the text.

Different ways of transferring proper nouns from one language into another have been put by Aguilera (2008) in another way:

1) Proper names can be copied or reproduced exactly as they are in the source text.

2) Proper names can be transcribed or transliterated or adapted on the level of spelling, phonology, etc.

3) Proper names can be substituted.

4) Proper names can be translated.

Some ways are more common than others. What is regarded as less common is the replacement of a source text common noun by a proper noun in the target text. All of the noted ways can be combined to produce new methods of transfer as well.

Personal names must not be translated as common nouns; they must be identified and then translated with regard to the target text. Acronyms are included in this area; some are translated while others are not. Hirschman and others (2001) distinguish between translation and transliteration and argue that the choice of transliteration or translation depends in part on convention. According to them, transliteration does not guarantee success in name recognition and sometimes translation makes the concept clear. In an attempt by Larkey and others (2003), they have concluded that a combination of static translation and transliteration provides a successful solution.

Aguilera's (2008) answer to the question 'should proper names be translated or not'? is that the macro and microstructures of each text will influence this issue; in story books personal names without any special meaning should not be altered but those with meaning have to be translated, since meaningful names play a role within a story and not translating them leads to the lack of fulfillment of intended communicative process.

Different ways of translating proper nouns are posed by some researchers in different studies, which express the choices a translator has for translating proper names regarding some factors such as the audience at different ages, the context in which a proper noun is used and some other factors. The table below represents Van Coillie's (2006) model of translating proper nouns: 
Table 1: Van Coillie's Model of Translating Proper Name (2006, p.123)

\begin{tabular}{|c|c|}
\hline Types of strategy & Character of translation \\
\hline 1) Reproduction & Leaving foreign names unchanged \\
\hline $\begin{array}{l}\text { 2) Nontranslation plus additional } \\
\text { explanation }\end{array}$ & $\begin{array}{l}\text { Adding explanations, either in the } \\
\text { form of a note or in the text itself }\end{array}$ \\
\hline $\begin{array}{l}\text { 3) Replacement of personal name } \\
\text { by a common noun }\end{array}$ & $\begin{array}{l}\text { Replacing a proper name by a } \\
\text { common noun that characterizes the } \\
\text { person }\end{array}$ \\
\hline $\begin{array}{l}\text { 4) Phonetic or morphological } \\
\text { adaptation to the target language }\end{array}$ & $\begin{array}{l}\text { Turning to phonetic transcription or } \\
\text { morphological adaptation }\end{array}$ \\
\hline 5) Exonym & $\begin{array}{l}\text { Replacing a name by a counterpart } \\
\text { in the target language }\end{array}$ \\
\hline $\begin{array}{l}\text { 6) Replacement by a more widely } \\
\text { known name from the source } \\
\text { culture or an internationally known } \\
\text { name with the same function }\end{array}$ & $\begin{array}{l}\text { Opting for recognize ability without } \\
\text { Abandoning the foreign context }\end{array}$ \\
\hline 7) Substitution & $\begin{array}{l}\text { Replacing a name by another name } \\
\text { from the target language }\end{array}$ \\
\hline $\begin{array}{l}\text { 8) Translation of names with a } \\
\text { particular } \\
\text { connotation }\end{array}$ & $\begin{array}{l}\text { Reproducing the connotation in the } \\
\text { target language, when names have } \\
\text { specific connotations }\end{array}$ \\
\hline $\begin{array}{l}\text { 9) Replacement by a name with } \\
\text { another or additional connotation }\end{array}$ & $\begin{array}{l}\text { Adding or changing the connotation } \\
\text { of a name }\end{array}$ \\
\hline 10) Deletion & Omitting all proper names \\
\hline
\end{tabular}

According to Ahanizadeh (2012), the strategy of reproduction is the most commonly used one in translating proper nouns from English into Persian.

Four general operations may take place in the translation process of proper nouns: transference, translation process, substitution or modification. It is possible that different translators use various strategies to deal with proper nouns and that these strategies entail different communicative effects for the respective audiences. In sum, translators may preserve all proper nouns in their original form or translate those which have equivalents in the target language and make the text more familiar to the readers.

\section{The problems on the way of the translation of the proper nouns}

Obstacles on the way of translation come from various sources because a word in a language reflects a unique experience of life. We are interested in the exact and actual meaning of the words of the source languages when translated in the target language. Proper nouns are exceptions in this case. The translation of proper names has been a challenging and problematic activity for every translator since many years ago. It cannot be simply found in dictionaries like other words.

Identification of proper names, according to Wacholder and others (1997) has two aspects: the recognition of the known names and the discovery of new names. It is sometimes difficult to determine the exact boundaries of proper nouns. They bring about ambiguity, for instance; sometimes it is not easy to distinguish the relationship between two nouns, whether a noun is a possessive name or a part of a name (Isreal's Shimon). Furthermore, they may be semantically ambiguous, whether a proper name is the name of a person, location or entity, like a car; or the United States is a geographical area or a political body which governs this area.

Familiarity with the target culture is a determining factor for readers, whether they can understand the proper noun posed by the translator or not. Whether a proper name can tell us the referent is a female or a male person (Mary-Tom, Alice - Sam), and geographical origin within the same language community depends on our familiarity with the culture in question, so proper nouns may be culturally specific and this feature leads to difficulty in their translation. Aguilera (2008) puts emphasis on "internationalism and multiculturalism" as factors influencing decision making in proper nouns translation. Even translation strategies are used by two different translators with different cultures differently.

Altogether, a series of factors, elements and circumstances must be taken into consideration before making a decision on the process of translation. One of the factors worth mentioning is age. Aguilera (2008:5) establishes the following classification of age:

1. pre-reading children (0 to 6 years old),

2. children capable of reading and writing (from 6 to adolescence), and 


\section{3. adolescents and youngsters.}

Books for children have always been in different languages and they have been translated into other languages in all over the world regarding internationalism and multiculturalism; children's literature must be translated with more care which means adaptation to the young reader's language is an important issue. This is true about the proper nouns as well. Translators must be aware of the realm of childhood adapting names to the target culture. Previous knowledge of the world influence perception and interpretation of the facts. Therefore, the lower the age, the less knowledge of the world a reader has. Readers' lack of background knowledge causes problems. As a result, a close attention must be given to the texts which are to be translated for young children.

The other problem with translation of the proper nouns is different grammatical rules and word order in different languages. In Chinese and Japanese languages, for instance, the order of a person's first and last name is the opposite to the Western order of first name and last name. Besides, sometimes in translating proper names there are many acceptable answers, as an example we refer to the study by Matthews (2007). He points out that English transliterations of Muhammad include Mohamad, Mohamed, Mohammad, Mohammed, Muhamad, Muhamed, Muhammad and Muhammed. Thus this variety makes the task more difficult for the translator. Losangeles and Lusangeles, and also, Washangton and Washington are possible Persian forms of two cities, Los Angeles and Washington, in the United States.

Another main problem posed by Nord (2003) is that proper nouns that exist in the same form both in the source and target culture cause some sort of problem, that is to say, when the given name is pronounced in a different culture, the character changes nationality and it may appear to the audience exotic. An English Richard, thus, turns into a German Richard, and a French Robert turns into an English Robert. The main point is that different nationalities may call the same character with different names; therefore using the same name to address them is a bit strange to the readers and brings about misconception. This may occur when the translator does not have sufficient knowledge about the target language pronunciation. In addition, a translator may not have enough knowledge about the source language and also about the conventions of pronouncing in the target language and just employs his/her own knowledge about the pronunciation of that particular language; at this point what takes place is the wrong pronunciation or misspelling by the translator. He/She may address it mistakenly employing a similar pronunciation of another language; for instance, addressing "Michael," /Maikel/ which is common in English instead of French "Mishel," while translating a French text into Persian. Therefore, translators' unfamiliarity with all aspects of that very language and its irregularities and exceptions leads to mistranslation by the translator and misunderstanding by the reader. To this respect, Hermjakob and others (2008) suggest creating new, plausible target language spellings that preserve the sounds of the source-language name as much as possible. A big problem of many authors and translators nowadays is that sometimes translators have just read some proper nouns in a particular language (not their own language) and are lack of knowledge about how to pronounce and spell them in their own language, in other words, they have no information about the conventions of some proper nouns pronunciation and spelling and their exceptions in their own language.

Abbreviations in a text in question, also, cause problems in proper name translation and close attention must be given to them (Hirschman and others, 2001). The reader of the translated text must be familiar with the abbreviations the translator uses from the source text, otherwise confusion and misconception will take place. If the abbreviations are not common in the target text, it is the translator's task to avoid using them in the original form. Instead they must be translated in such a way which is understood by the reader.

In addition to all these problems, a point worth noting is that some proper names need updates when stylistic conventions change like the names of softwares. Thus if the translator uses the old names confusion may occur for the reader. Larkey and others (2003) suggest IR tasks involving news corpora, like TDT and TREC cross language. These corpora have proper names at their core, but according to these authors, they often do not contain names, numbers, technical terms, and acronyms. Their translations may not reflect current usage.

Muhammad and others (2007) state that listing proper nouns in the dictionary, which may be considered as a solution, creates many problems. According to them, it is a tedious and time-consuming task and this solution is absolutely inefficient since the dictionary with all proper nouns slows down the process of translation. Besides, it is impossible to insert all the proper nouns from different cultures and linguistic knowledge in dictionaries. Proper nouns are unlimited in number and have many kinds and varieties from country to country and culture to culture.

In an investigation done by Hermjakob and others (2008), it is found that machine translation (MT), which is suggested by some researchers to solve the problem of proper nouns translation, is not effective, particularly across languages with different sound systems. It may mistake an unknown name for a common noun; the following procedure is posed by them:

"The simplest way to integrate name handling into SMT is:

(1) run a named-entity identification system on the source sentence,

(2) transliterate identified entities with a special-purpose transliteration component, and

(3) run the SMT system on the source sentence, as usual, but when looking up phrasal translations for the words identified in step 1, instead use the transliterations from step 2" (p. 390).

Elsewhere (on the same page) in their article they argue that this procedure will not work for some reasons:

- "Automatic named-entity identification makes errors. Some words and phrases that should not be transliterated are nonetheless sent to the transliteration component, which returns a bad translation. 
Not all named entities should be transliterated. Many named entities require a mix of transliteration and translation. /jnub kalyfurnya/ "Southern California," the first Arabic word is translated, and the second word is transliterated.

Transliteration components make errors. The base SMT system may translate a commonly occurring name just fine,

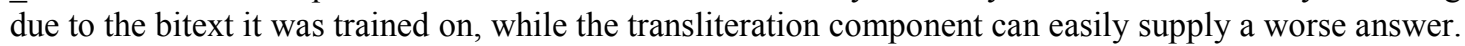

- Integration hobbles SMT's use of longer phrases. Even if the named-entity identification and transliteration components operate perfectly, adopting their translations means that the SMT system may no longer have access to longer phrases that include the name".

Chen and others (2001) reject MT as an appropriate set for translating proper names. They proved that in MT, since proper names are infrequent relative to other words in corpora, they may be neglected as unimportant words. The problems mostly occur in languages with different alphabetic systems. Since each alphabetic system has its own rules, these problems may take place in the translation of proper nouns. In spite of this fact, no translation is free from such problems, and errors may occur in translating two languages with the same alphabetic system as well. Persian is a language which has phonetic limitations and thus translation problems may occur a lot in translating other languages to this language, thus it may result in difficulties and complications for the translator.

Different problems of translating proper nouns have been noted so far. Communication in the world today is of key importance so solving these complications and problems is crucial; how to solve these difficulties and create a pure translation is a point which has garnered translators' attention.

\section{Possible solutions for the translation of proper names}

According to Jaleniauskienè and Čičelyte (2009), there are two general translation strategies, foreignization (literal translation), in which all the cultural elements will be preserved, and domestication, in which adaptation will take place and cultural elements will be left. Of course the issues of foreignization and domestication have been termed by many scholars differently. A variety of translation strategies have been proposed by different translation theorists such as Davies (2003). She counts seven strategies: preservation, addition, omission, globalization, localization, transformation and creation; each is discussed below:

Preservation: (literal or direct translation) occurs when a translator provides no further explanation.

Addition: when a translator preserves the original item but provides necessary information (additional information can be provided within the text or in a footnote).

Omission: is the opposite strategy to addition when translators omit a problematic case so that no trace of it is found. Davies (2003:80) argues that, when the inclusion of a problematic culture-specific item might create a confusing or inconsistent effect, it is better to be omitted.

Globalization: is replacing culture-specific references with general ones. This strategy is a form of domestication. For example, a particular breed of a cat is translated simply as "a cat".

Localization: when translators try to "anchor a reference firmly". This strategy includes phonological and grammatical adaptation of names and it occurs when translators regard the culture of the target audience in translation, in other words they try to make the translation closer to the target language. According to Davies, this process is a kind of distortion of the original.

The last strategy, creation, is the making of an item which is totally different from the source language. This strategy is a kind of compensation.

Her categorization of proper noun translation strategies is illustrated in the following figure:

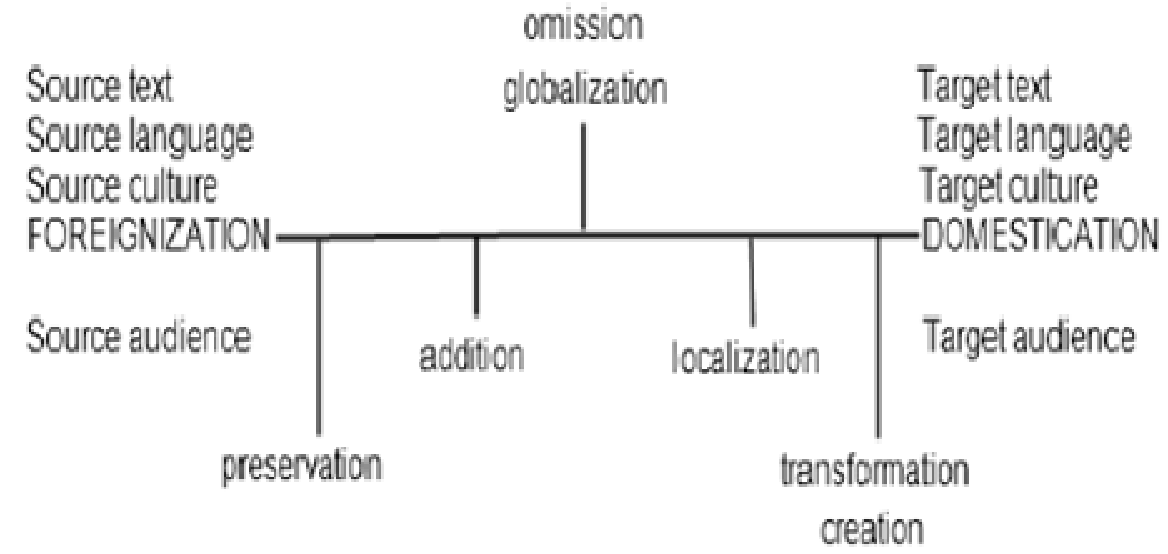

Figure1. A continuum between foreignization and domestication, (Davies, 2003)

Jaleniauskienè and Čičelyte (2009) believe that the strategy which is very close to foreignization is preservation because the item is not changed. By contrast, creation is close to domestication. Globalization and omission are between foreignization and domestication, Addition, on the other hand, is closer to foreignization, because it reminds the reader that it is really a foreign text and finally localization is closer to domestication, because the translator, according to the rules of the target language, makes adaptation. 
Sadeghi Ghadi (2010) is another researcher who has mentioned a number of ways in which proper names can be translated:

"1. They can be imported unchanged from the SL text;

2. They can be modified to fit the

phonological/graphological system of the TL. This, of course, is something that has or has not been done for the translator by his/her speech community in the case of conventional place names like Prague, the Hague, Rome and so on;

3. They can be expanded with a gloss to make up for the TL reader's lack of world knowledge in the target culture;

4. On occasion, they might be omitted altogether (perhaps replaced with a paraphrase) if considered peripheral in terms of the central message of the text or if retaining them would be more likely to cause the reader to pause in puzzlement. True, this might happen in interpretation, but could not be ruled out altogether in translation, either;

5. In rare cases, they might even be introduced in the TL text where, instead of a proper noun, the SL text contains a cultural allusion unlikely to be understood by the TL reader (p.3)".

Hirschman and others (2001: 24) likewise distinguish five different ways of treating names:

a) Simple transcription and translation,

b) Clarification (e.g., adding contextual information),

c) Simplification (e.g., acronyms) and

d) Omission (e.g., using anaphora).

In their study they indicated that transcription and translation are applied more than other categories. The actual choice of strategy the translator makes depends on a variety of factors. Van Coillie (2006) introduced some of them:

Table 2.Van Coillie's categorization of translator motives (2006, p.129)

\begin{tabular}{ll}
\hline factors & Translator Motives \\
\hline Nature of the name & $\begin{array}{l}\text { The connotation attached to a name } \\
\text { appears to be the most important reason } \\
\text { for changing it. }\end{array}$ \\
Textual factors & $\begin{array}{l}\text { Names are invariably embedded in a } \\
\text { cultural context. If the context is not } \\
\text { developed to a substantial degree, the } \\
\text { chances are that the corresponding } \\
\text { names will be modified. }\end{array}$ \\
The translator's frame of reference & $\begin{array}{l}\text { In making their choices, translators are } \\
\text { guided by their own frame of reference, } \\
\text { the total sum of their knowledge, } \\
\text { experiences, ideas, norms and values. }\end{array}$ \\
Other factors & $\begin{array}{l}\text { Whatever strategy the translator chooses, } \\
\text { sometimes the ultimate choices are made } \\
\text { by or in co-operation with other factors } \\
\text { in the literary communication process. }\end{array}$ \\
\hline
\end{tabular}

Ahanizadeh (2012) introduced samples of strategies used in translating proper nouns (English into Persian). Some of them worth mentioning are posed below:

a) Reproduction:

\section{Jack: جك}

b) Phonetic or morphological adaptation to the target language

Smith: اسميت

c) Exonym:

Damascus: دمثق 
Names of countries do not cause problem to the translator (Abdolmaleki, 2012). According to him, they do not have connotations. But sometimes using a name of a place denotes more than that very name. For instance, in the clause 'Zire be Kerman bordan' in Persian, which means 'to carry coal to the Newcastle', Kerman must not be translated as a name of a city, but the metaphoric meaning of that must be taken into consideration. Metaphoric meaning is important not the literal one. Names of countries, in different nationalities, may differ not only in pronunciation or spelling, but also with respect to morphology. It is the translator's task to identify these differences. There are lists of the official names of countries in Persian and English that can be consulted. Of course, there are proper names, particularly the names of the countries that exist in the same form both in the source and the target culture.

The names of the streets, hospitals and schools are not translated since the author intends to introduce them in the source language culture not describing their characteristics. There is no need to translate the names of the brands, because they are known to the target language as well.

As mentioned before there are different acceptable forms of some names in a language such as Los Angeles and Washington. If the translator is doubtful about which one of them to choose, he/she should select the one which is closer to the source language in pronunciation. Also, sometimes there are some names the translation of which has been common in a language in particular forms which are not correct regarding the phonological rules or other conventions, e.g. Thomas which is common as /Toomas/ in Persian, not/taames/, a correct and precise pronunciation. Such names have been used in forms which are not correct since many years ago and it is not necessary to change them.

What Wacholder and others (1997) believe is that capitalization is a factor helps the reader to distinguish between a common name and a proper name, for instance, candy as the name of food versus Candy, a name for a person; or the house which refers to a building versus the House as an organization. However, capitalization does not always work because what constitutes a name is not always clear to help us distinguish a name from a non-name. As an example, Monday may show time as a common noun or be an indication of a proper name. If a sentence initial name in capital letter appears capitalized elsewhere in the sentence, it is a proper noun. As a matter of fact capitalization still needs more consideration and discoveries.

Different researches have different suggestions for appropriate translation of proper nouns. Muhammad and others (2007) propose machine translation (MT) in order to find a solution for handling the proper nouns. Also Hirschman and others (2001) put emphasis on machine translation as a solution to proper nouns translation and believe that MT has brought breakthroughs in technology of translation. In a study by Shia and others (2007), they used mixed-syllablemapping model and hybrid translation extraction method to translate proper names. Virga and Khudanpur (2003) used cross-lingual information retrieval (CLIR) method in this regard. Chen and others (2001) in their study indicat that massage understanding conference (MUC) and multilingual entity task are used for person name, location and time recognition.

In sum, if translators face problems in translating proper names, the best way is to refer to acceptable and trustable references; that is to say, they do not have to trust their own knowledge or refer to the references they are not sure about but they must make sure that what they employ in their translation is acceptable in the target language. In so doing, referring to acceptable and trustable references is suggested. Now the question is 'what are these acceptable and trustable references'? The answer is the ones which are able to introduce the rules, conventions and exceptions. Good dictionaries help a lot in this matter.

Translators do all sorts of ways to make proper names understood by the readers: non-translation, transcription or transliteration, morphological adaptation to the target language, cultural adaptation, substitution, and so on. Also, they do not always use the same techniques with all the proper names of a particular text that they are translating. But the point is that successful translators take different factors into account in order to solve proper nouns translation problem which results in better communication.

\section{Conclusion}

Since proper nouns usually have various allusions, their translation is complicated. They hold an exceptional position with regard to the language system. Thus, the translation of these nouns is a challenge for translators. In this regard Sadeghi Ghadi (2010) states: "translation of proper names is not a trivial issue but, on the contrary, may involve a rather delicate decision-making process, requiring on the part of the translator careful consideration of the meanings the name has before deciding how best to render it in the target language" (p.v1). Abdolmaleki (2012), also, believes that proper nouns are beyond language and may be found in encyclopedia not dictionaries. So, translating proper nouns is not that simple. Taking all the discussed points into consideration and giving closer attention to the nature of the proper nouns, we come to this conclusion that even if proper nouns are non-descriptive, they are informative. In fictional texts there is no name that has no informative function at all, however subtle it may be. Proper nouns, beyond their identifying function, may also carry meaning.

There is no flexibility about how to translate a name. There are no rules for the translation of proper nouns. Translators do not always use the same techniques with all the proper nouns of a particular text that they are translating. A main criterion for the translation is making this identifying function work for the target audience. Translators do various sorts of things with proper nouns. Procedures that could be applied for rendering proper nouns manipulated in many ways. In so doing, different factors must be taken into consideration to come to the best strategy to use in a particular text. The most salient factor, as noted before, is the culture of the readers. Different languages, thoughts and views exist as a consequence of multiculturalism. Different proper nouns are translated differently in various cultures. Even two 
different groups of translators, from different cultures, might approach the same work using opposed translation strategies. The audience with different cultural and linguistic knowledge cannot have the same associations. Therefore, it is concluded that for a translator to translate efficiently it is not sufficient to have a good command of linguistics. Cultural sensitivity is also an important factor. Moreover, a translator has to consider the intended audience and their age, as different audiences require different translation strategies; knowledge of the target culture, experiences, ideas, and values help translators to make the best choice among different ways of treating proper nouns. Thus as Al-Hassan and Jordan (2013) believe, translations not only include linguistic content but also values, norms and ways of life in a given culture. Indeed, the goal of translation is to present the target language, both linguistically and culturally.

On the whole, it is impossible to achieve absolute equivalence due to specific aspects in the languages but what the translators do in this process is trying to preserve authentic effect achieved by the names and to improve transliteration accuracy. They try, when writing foreign names in their own language, to preserve the way they sound and by applying different translation strategies they find appropriate ways to render proper nouns. Selecting appropriate ways has advantages; one of which, according to Larkey and others (2003), is that solutions to the problem of nouns translation may even be effective for translating other unknown words. Also, as Chen and others (2001) believe, if translators try to have correct translation, the cost of developing a large scale dictionary for proper names will be reduced. In spite of some limitations of machine translation (MT), handling proper nouns using MT has caused too much success to date and this method is suggested by many researchers.

Altogether, special attention is needed to be paid to the translation of proper nouns since it is a real challenge for all translators. Proper nouns are loaded with semantic content in the source context and there are a number of considerations of how this content may be preserved in the target communication situation which are critically essential. Considerations include elements like the specific audience, intertextual relationships and translation norms, in consistency with the principle of relevance. Translators need a good knowledge of the background of their target audience. In addition, they must have sufficient knowledge about the content that they translate otherwise they encounter difficulties. If the original meaning changes, the intended massage by the writer may not be conveyed.

Good experience and knowledge is needed to translate a text precisely; any inconsistencies or other problems of the foundation can cause the whole translation to crumble. To this respect, translators are recommended to give close attention to different aspects of translation and particularly proper nouns. A good translator has to add pieces of information from his/her own repertoire of the target language knowledge to convey what the text means.

\section{References}

Abdolmaleki, S.D. (2012). Proper Names in Translation: an Explanatory Attempt. The Social Sciences, 7(6), 832-837.

Aguilera, E.C. (2008). The Translation of Proper Names in Children’s Literature. E-f@bulations / E-f@bulações, 2, 110.

Ahanizadeh, S. (2012).Ttranslation of Proper Names in Children's Literature. Journal of Language and Translation, 3(1), 61-71.

Algeo, J. (1973). On Defining the Proper Name. Gainesville: University of Florida Press.

Al-Hassan, A., \& Jordan, A. (2013). The importance of Culture: Should culture be translated? International Journal of Applied Linguistics \& English Literature, 2 (2), 96-100.

Anabisarab, M. (2009). Proper Names Translation in Translated Fictions for Adolescents from English to Persian. Journal of Translation studies, 7(28), 9-30.

Bloom, P. (2001). How Children Learn the Meaning of Words. Behavioral and Brain Sciences, 24, 1095-1103.

Chen, H., Huang, S.J., Ding, Y.W., \& Tsai, S.C. (2001). Proper Name Translation in Cross-Language Information Retrieval. COLING '98 Proceedings of the 17th International Conference on Computational Linguistics, (1), 232-236.

Davies, E.E., (2003). A Goblin or a Dirty Nose? The Translator: Studies in Intercultural Communication, 9 (1), 65-100.

Fernandes, L. (2006). Translation of Names in Children's Fantasy Literature: Bringing the Young Reader into Play. New Voices in Translation Studies, 2, 44-57.

Hall, D.G. (1999). Semantics and the Acquisition of Proper Names. In Language, Logic, and Concepts. Cambridge: Cambridge University Press.

Hermjakob, U., Knight, K., \& Daum'e, H. (2008). Name Translation in Statistical Machine Translation Learning When to Transliterate. Proceedings of ACL-08: HLT, International Conference on Computational Linguistics, 389-397.

Hirschman, L., Reeder, F., Burger, J.D., \& Miller, K. (2001). Name Translation as a Machine Translation Evaluation Task. [Online]. 2, 21-28.

Jaleniauskienė, E., \& Čičelytė, V. (2009). The Strategies for Translating Proper Names in Children's Literature. Studies about languages, 15, 1648-2824.

Macnamara, J, (1982). Names for Things. A Study of Human Learning. Cambridge: Cambridge University Press.

Larkey, L.S., AbdulJaleel, N., \& Connell, M. (2003).What's in a Name? Proper Names in Arabic Cross Language Information Retrieval. Available at: http://Ciir.cs.umass.edupenynsu. 
Levinaz, E. ( 2003). Proper Names. Stanford: Stanford University Press.

Longobardi, G. (1994). Reference and Proper Names: A Theory of N-Movement in Syntax and Logical Form. Linguistic Inquiry, 25(4), 609-665.

Matthews, D. (2007). Machine Transliteration of Proper Names. Edinburgh: Edinburgh University Press.

Muhammad, U., Khan, A., Khan. M.N., Ayyub, K. \& Muhammad, S. (2007). Handling Proper Nouns in Machine Translation from English into Urdu. Journal of Information \& Communication Technology, 1(2), 56-64.

Nord, C. (2003). Proper Names in Translations for Children. Alice in Wonderland as a Case in Point. Available at: http://id.erudit.org/iderudit/006966ar, 48, 182-196.

Quirk, R., Greenbaum, S., Leech, G., \& Svartvik, J. (1972). A Grammar of Contemporary English. Singapore: Longman Group Ltd.

Sadeghi Ghadi, A. (2010). Translation of Proper Names. Available at: Translation Directory.com.

Sciarone, B. (2008). Proper Names and Meaning. Studia Linguistica, 21(2), 73-86.

Shia, M.S., Lin, J.H., Yu, S., \& Lu, W.H. (2007). Improving Translation of Unknown Proper Names Using a Hybrid Web-based Translation Extraction Method. Available at: http://www.aclclp.org.tw.

Sperber, D., \& Wilson, D. (1986). Relevance. Oxford: Basil Blackwell.

Van Coillie, J. (2006). Character Names in Translation: A Functional Approach. In J.Van Coillie, \& W. P Verschueren (Eds.), Children's Literature in Translation: Challenges and Strategies (pp. 123-139). Manchester \& Kinderhook: St. Jerome Publishing.

Virga, P. \& Khudanpur, S. (2003). Transliteration of Proper Names in Cross-Lingual Information Retrieval. ACL 2003 workshop MLNER, 57-64.

Vermes, A.P. (2003). Proper Names in Translation: an Explanatory Attempt. Across Languages and cultures 4(1), 89108.

Wacholder, N., Ravin, Y., \& Choi, M. (1997). Disambiguation of Proper Names in Text. Published in: ANLC '97 Proceedings of the Fifth Conference on Applied Natural Language Processing, 202-208. 